\title{
Conserved currents in the Palatini formulation of general relativity
}

\author{
Glenn Barnich* \\ Physique Théorique et Mathématique \\ Université libre de Bruxelles and International Solvay Institutes \\ Campus Plaine C.P. 231, B-1050 Bruxelles, Belgium \\ E-mail: gbarnicheulb.ac.be

\section{Pujian Mao} \\ Center for Joint Quantum Studies and Department of Physics \\ School of Science, Tianjin University \\ 135 Yaguan Road, Tianjin 300350, P. R. China \\ E-mail: pjmao@tju.edu.cn

\section{Romain Ruzziconi} \\ Physique Théorique et Mathématique \\ Université libre de Bruxelles and International Solvay Institutes \\ Campus Plaine C.P. 231, B-1050 Bruxelles, Belgium \\ E-mail: rruzzico@ulb.ac.be
}

We derive the expressions for the local, on-shell closed co-dimension 2 forms in the Palatini formulation of general relativity and explicitly show their on-shell equivalence to those of the metric formulation. When compared to other first order formulations, two subtleties have to be addressed during the construction: off-shell non-metricity and the fact that the transformation of the connection under infinitesimal diffeomorphisms involves second order derivatives of the associated vector fields.

Corfu Summer Institute 2019 "School and Workshops on Elementary Particle Physics and Gravity" (CORFU2019)

31 August - 25 September 2019

Corfu, Greece

${ }^{*}$ Speaker. 


\section{Introduction}

The Palatini first order formulation is a convenient starting point for the standard Hamiltonian approach to general relativity by Arnowitt, Deser and Misner [1-3]. It is in this framework that appropriate surface integrals at spatial infinity for energy-momentum have originally been constructed $[4,5]$ and that the Hamiltonian formulation is presented in $[6,7]$.

Conserved quantities in first order formulations of general relativity have recently been investigated from a variety of perspectives, see e.g. [8-19]. In the approach that we follow here [20-22], one constructs conserved co-dimension 2 forms in the linearized theory from the weakly vanishing Noether currents associated to gauge symmetries. Indeed, one can show in the linearized theory that there are conserved co-dimension 2 forms for each reducibility parameter of the background. The latter correspond to the Killing vectors of the background metric in general relativity and one can show that there are no other conserved co-dimension 2 forms which are non-trivial. The method has been applied recently to first order formulations of general relativity where the variables are either a vielbein and a Lorentz connection in coordinate basis [23], or a vielbein and the spin coefficients of the Newman-Penrose formalism [24]. Two additional general results have been added in that context: a general expression for conserved co-dimension 2 forms applicable in a generic first order theory and a detailed discussion of the breaking term, the flux terms that appear on the right hand side of what would be a conservation law when one uses general gauge parameters rather than reducibility parameters of the background.

Two subtleties have to be faced when applying this construction to the Palatini formulation of general relativity. The first is that the theory is not first order in the sense that the transformation of the connection under infinitesimal diffeomorphisms involves second order derivatives of the vector field parametrizing these diffeomorphisms. This leads to a weakly vanishing Noether current that is not first order. It turns out however that all higher order terms are contained in a total derivative and such terms are easily handled by the contracting homotopy operator used to built the co-dimension 2 forms. As a consequence, the construction is as straightforward as in other first order approaches to general relativity. The second subtlety is that, as for other discussions of symmetries on the level of an action principle, all computations are performed off-shell. For the Palatini formalism, this means that one has to deal with non-metricity. Closely related considerations in theories going beyond general relativity can be found in [25].

The paper is organized as follows. We start with a very brief review of how to construct conserved co-dimension 2 forms out of weakly vanishing Noether currents. More details can be found in the original literature cited above and an extensive recent summary has been provided in [24]. We then discuss various identities satisfied by the curvature tensor in the general context of a nonholonomic frame including torsion and non-metricity because these are relevant for the Noether identities that are crucial to the construction. We then apply these general considerations to the particular case of the Palatini formulation for which one uses a coordinate basis and a connection without torsion. Finally we construct the co-dimension 2 forms and the associated breaking terms. 


\section{Construction of co-dimension 2 forms}

\subsection{General case}

We consider a theory with a Lagrangian $n$-form $\mathscr{L}=L d^{n} x$ in $n$ dimensional spacetime. The fields of the variational principle are denoted by $\phi^{i}$. Consider a generating set (see e.g. [26], chapter 3) of non trivial gauge transformations $\delta_{\varepsilon} \phi^{i}=R_{\alpha}^{i}\left(\varepsilon^{\alpha}\right)$. One can prove that there is an isomorphism between equivalence classes of on-shell closed co-dimension 2 forms and equivalence classes of reducibility parameters $\bar{f}^{\alpha}[x, \phi]$ satisfying $R_{\alpha}^{i}\left(\bar{f}^{\alpha}\right) \approx 0$. Equivalent co-dimension 2 forms differ on-shell by an exact local form while equivalent sets of reducibility parameters agree on-shell.

The relation between on-shell closed co-dimension 2 forms and reducibility parameters is constructive. For arbitrary gauge parameters $f^{\alpha}$, a direct application of the Leibniz rule for total derivatives leads to

$$
R_{\alpha}^{i}\left(f^{\alpha}\right) \frac{\delta \mathscr{L}}{\delta \phi^{i}}=f^{\alpha} R_{\alpha}^{+i}\left(\frac{\delta \mathscr{L}}{\delta \phi^{i}}\right)+d_{H} S_{f}
$$

for some weakly vanishing $n-1$ form

$$
S_{f}=S_{\alpha}^{i \mu}\left(\frac{\partial}{\partial d x^{\mu}} \frac{\delta \mathscr{L}}{\delta \phi^{i}}, f^{\alpha}\right)
$$

The $n-2$ form can be constructed by using the contracting homotopy $\rho_{H}$ for the horizontal differential of the variational bi-complex $[27,28]$

$$
\left\{d_{H}, \rho_{H}\right\} \omega^{p}=\omega^{p} \text { for } p<n
$$

Indeed, the Noether identities that are associated to the generating set of non-trivial gauge transformations are

$$
R_{\alpha}^{+i}\left(\frac{\delta \mathscr{L}}{\delta \phi^{i}}\right)=0
$$

For reducibility parameters $\bar{f}^{\alpha},(2.1)$ reduces to $d_{H} S_{\bar{f}} \approx 0$. One then shows (see section 3.3 of [20] for details) that the weakly vanishing terms on the right hand side can be absorbed by the horizontal differential of a "doubly" weakly vanishing $n-1$ form $M_{\bar{f}}$ on the left hand side, leading to $d_{H}\left(S_{\bar{f}}+M_{\bar{f}}\right)=0$. When applying the contracting homotopy to $J_{\bar{f}}=S_{\bar{f}}+M_{\bar{f}}$,

$$
k_{\bar{f}}=\rho_{H} J_{\bar{f}}
$$

it then follows from (2.3) that

$$
d_{H} k_{\bar{f}}=J_{\bar{f}} \approx 0 .
$$

In case where one can show that a set of reducibility parameters is equivalent to a set for which $R_{\alpha}^{i}\left(\bar{f}^{\alpha}\right)=0$, the reasoning simplifies since in this case $d_{H} S_{\bar{f}}=0$, and the application of (2.3) now directly yields $d_{H} k_{\bar{f}}=S_{\bar{f}} \approx 0$ with $k_{\bar{f}}=\rho_{H} S_{\bar{f}}$. Similarily, in linear gauge theories, the application of the homotopy formula to $M_{\bar{f}}$ gives rise to a weakly vanishing and thus trivial $n-2$ form, which can be omitted. In this case, we still have $k_{\bar{f}}=\rho_{H} S_{\bar{f}}$ but now $d_{H} k_{\bar{f}} \approx S_{\bar{f}} \approx 0$. 


\subsection{Linearized theories and asymptotics}

For the purposes of exposition, we focus on the Einstein-Hilbert action in metric formulation, where a generating set of gauge transformations corresponds to the Lie derivative of the metric, $\delta_{\xi} g_{\mu \nu}=\mathscr{L}_{\xi} g_{\mu \nu}$. In spacetime dimensions $n \geq 3$, one can then show that all equivalence classes of reducibility parameters admit representatives $\xi^{\rho}[x]$ that do not depend on $g_{\mu v}$ and its derivatives. The condition that such vectors are reducibility parameters then reduces to the Killing equation for a generic metric. Since a generic metric does not have Killing vectors, no non-trivial conserved $n-2$ forms can be constructed in general relativity. However, one can linearize the theory around a background solution $\bar{g}_{\mu \nu}$. A generating set of gauge transformations of the linearized theory corresponds to the Lie derivative of the background metric, $\delta_{\xi} h_{\mu v}=\mathscr{L}_{\xi} \bar{g}_{\mu \nu}$. It then follows that there are as many conserved $n-2$ forms as there are Killing vectors of the background solution. The explicit expressions of the $n-2$ forms are obtained by applying the construction described in previous subsection in the framework of the linearized theory. This has been done explicitly for Einstein gravity in [20].

More generally, one can show [21] that the $n-2$ forms of the linearized theory can be obtained from the weakly vanishing co-dimension 1 form $S_{f}$ of the full theory through

$$
k_{f}[\delta \phi, \phi]=k_{f}^{\mu v}\left(d^{n-2} x\right)_{\mu v}=\frac{|\lambda|+1}{|\lambda|+2} \partial_{(\lambda)}\left[\delta \phi^{i} \frac{\delta}{\delta \phi_{((\lambda) v)}^{i}} \frac{\partial}{\partial d x^{v}} S_{f}\right],
$$

by replacing $f$ by reducibility parameters of the linearized theory, $\phi^{i}$ by the background solution $\bar{\phi}^{i}$ and $\delta \phi^{i}$ by any solution $\bar{\varphi}^{i}$ of the theory linearized around $\bar{\phi}^{i}$. We refer to [27] and [28] for the explicit expressions for the higher order Euler-Lagrange derivatives. Our conventions and notations for multi-indices are summarized in the appendix of [20].

For theories such as general relativity in metric formulation where $S_{f}$ is at most of second order in derivatives, the formula involves the higher order Euler-Lagrange operators only up to order 2 and reduces to

$$
k_{f}[\delta \phi, \phi]=\frac{1}{2} \delta \phi^{i} \frac{\delta}{\delta \phi_{v}^{i}} \frac{\partial}{\partial d x^{v}} S_{f}+\frac{2}{3} \partial_{\sigma}\left[\delta \phi^{i} \frac{\delta}{\delta \phi_{v \sigma}^{i}} \frac{\partial}{\partial d x^{v}} S_{f}\right] .
$$

For later use, note that for a local function $M$ involving the fields and their derivatives up to some finite order, a key property of the higher order Euler-Lagrange derivatives is that they "absorb" total derivatives,

$$
\frac{\delta \partial_{\lambda} M}{\delta \phi^{i}}=0, \quad \frac{\delta \partial_{\lambda} M}{\delta \phi_{v}^{i}}=\delta_{\lambda}^{v} \frac{\delta M}{\delta \phi^{i}}, \quad \frac{\delta \partial_{\lambda} M}{\delta \phi_{\mu v}^{i}}=\delta_{\lambda}^{(\mu} \frac{\delta M}{\delta \phi_{v)}^{i}},
$$

where the round (square) brackets denote (anti) symmetrization of enclosed indices divided by the factorial of the number of indices involved. Furthermore, if $M^{1}$ depends at most on first order derivatives, the Euler-Lagrange derivatives of order one reduce to partial derivatives,

$$
\frac{\delta M^{1}}{\delta \phi_{v}^{i}}=\frac{\partial M^{1}}{\partial \phi_{v}^{i}} .
$$

As shown in detail in [24], when using general gauge parameters $f^{\alpha}$ instead of reducibility parameters, non-conservation is controlled by the co-dimension 1 form $b\left[\delta \phi, R_{f}, \phi\right]$ defined by

$$
b=-\partial_{(\lambda)}\left[R_{\alpha}^{i}\left(f^{\alpha}\right) \delta \phi^{j} \frac{\delta}{\delta \phi_{(\lambda) v}^{j}} \frac{\partial}{\partial d x^{v}}\left(\frac{\delta \mathscr{L}}{\delta \phi^{i}}\right)\right]
$$


which satisfies $b\left[\delta \phi, R_{f}, \phi\right]=-b\left[R_{f}, \delta \phi, \phi\right]$ by construction. Indeed, when $\phi^{i}$ is a solution to the equations of motion, $\delta \phi^{i}$ a solution to the linearized equations of motion, the co-dimension 2 form $k_{f}$ constructed as in (2.7) is is no longer $d_{H}$-closed but satisfies instead

$$
d_{H} k_{f}=b .
$$

As in asymptotically flat general relativity at null infinity [29-31], these on-shell non-closed codimension 2 forms $k_{f}$ are in general not integrable either.

\section{Vielbeins and connection}

Now, we recall some notions of vielbeins and connection by including torsion and non-metricity into the standard discussion. In particular, this completes the results of $[23,24]$ by considering nonmetricity.

\subsection{General case}

Consider an $n$-dimensional spacetime with a moving frame (or vielbein)

$$
e_{a}=e_{a}{ }^{\mu} \frac{\partial}{\partial x^{\mu}}, \quad e^{a}=e^{a}{ }_{\mu} d x^{\mu},
$$

where $e_{a}{ }^{\mu} e^{a}{ }_{v}=\delta_{v}^{\mu}, e_{a}{ }^{\mu} e^{b}{ }_{\mu}=\delta_{a}^{b}$, and $\partial_{a} f=e_{a}(f)$. The structure functions are defined by

$$
\left[e_{a}, e_{b}\right]=D_{a b}^{c} e_{c} \Longleftrightarrow d e^{a}=-\frac{1}{2} D^{a}{ }_{b c} e^{b} e^{c} .
$$

For further use, note that if $\mathbf{e}=\operatorname{det} e^{a}{ }_{\mu}$, then

$$
\partial_{\mu}\left(\mathbf{e} e_{a}^{\mu}\right)=\mathbf{e} D_{b a}^{b}
$$

and, if we define,

$$
d_{b c}^{a}=e_{\lambda}^{a} \partial_{b} e_{c}^{\lambda}
$$

then

$$
d_{\rho \mu}^{\sigma}=-e_{d}{ }^{\sigma} \partial_{\rho} e_{\mu}^{d}, \quad D_{b c}^{a}=2 d_{[b c]}^{a},
$$

where it is understood that tangent space indices $a, b, \ldots$ and world-indices $\mu, v, \ldots$ are transformed into each other by using the vielbeins and their inverse.

In addition, we assume that there is an affine connection

$$
D_{a} e_{b}=\Gamma_{b a}^{c} e_{c} \Longleftrightarrow D_{b} v^{a}=\partial_{b} v^{a}+\Gamma_{c b}^{a} v^{c} .
$$

The components of the torsion tensor are given by

$$
\begin{gathered}
T_{\mu \nu}^{a}=\partial_{\mu} e^{a}{ }_{\nu}-\partial_{\nu} e^{a}{ }_{\mu}+\Gamma_{b \mu}^{a} e^{b}{ }_{\nu}-\Gamma_{b v}^{a} e_{\mu}^{b}, \\
T_{a b}^{c}=2 \Gamma_{[b a]}^{c}+D_{b a}^{c}=2\left(\Gamma_{[b a]}^{c}+d^{c}{ }_{[b a]}\right),
\end{gathered}
$$

while the components of the curvature tensor can be written as

$$
R^{f}{ }_{c \mu v}=\partial_{\mu} \Gamma^{f}{ }_{c v}-\partial_{v} \Gamma^{f}{ }_{c \mu}+\Gamma^{f}{ }_{d \mu} \Gamma^{d}{ }_{c v}-\Gamma^{f}{ }_{d v} \Gamma^{d}{ }_{c \mu},
$$




$$
R^{f}{ }_{c a b}=\partial_{a} \Gamma_{c b}^{f}-\partial_{b} \Gamma_{c a}^{f}+\Gamma^{f}{ }_{d a} \Gamma_{c b}^{d}-\Gamma^{f}{ }_{d b} \Gamma_{c a}^{d}-D^{d}{ }_{a b} \Gamma^{f}{ }_{c d}
$$

Furthermore,

$$
\left[D_{a}, D_{b}\right] v_{c}=-R_{c a b}^{d} v_{d}-T_{a b}^{d} D_{d} v_{c} .
$$

The Bianchi identities are given explicitly by

$$
R_{[b c d]}^{a}=D_{[b} T_{c d]}^{a}+T_{f[b}^{a} T_{c d]}^{f}, \quad D_{[f} R_{|b| c d]}^{a}=-R_{b g[f}^{a} T_{c d]}^{g},
$$

where a bar encloses indices that are not involved in the (anti) symmetrization. The Ricci tensor is defined by $R_{a b}=R_{a c b}^{c}$, while $S_{a b}=R_{c a b}^{c}$. Contracting the Bianchi identities gives

$$
\begin{gathered}
R_{a b}-R_{b a}=S_{a b}-D_{c} T^{c}{ }_{a b}-2 D_{[a} T_{b] c}^{c}-T^{c}{ }_{d c} T^{d}{ }_{a b}, \\
2 D_{[f} R_{|b| d]}+D_{c} R_{b d f}^{c}=R_{b g} T^{g}{ }_{d f}-2 R_{b[f|g|}^{c} T_{d] c}^{g}, \\
D_{[f} S_{c d]}=-S_{g[f} T^{g}{ }_{c d]} .
\end{gathered}
$$

Assume now that there is a pseudo-Riemannian metric,

$$
g_{\mu \nu}=e^{a}{ }_{\mu} g_{a b} e^{b}{ }_{v}
$$

i.e., a symmetric, non-degenerate 2-tensor. As usual, tangent space indices $a, b, \ldots$ and world indices $\mu, v, \ldots$ are lowered and raised with $g_{a b}, g_{\mu v}$, and their inverses. The non-metricity tensor is defined as $\Xi^{a b}=d g^{a b}+2 \Gamma^{(a b)}$. The associated Bianchi identities are given by $d \Xi^{a b}+\Gamma_{c}^{a} \Xi^{c b}+$ $\Gamma_{c}^{b} \Xi^{a c}=2 R^{(a b)}$. More explicitly,

$$
\Xi_{c}^{a b}=D_{c} g^{a b}, \quad 2 D_{[c} \Xi^{a b}{ }_{d]}=-\Xi_{f}^{a b} T_{c d}^{f}+2 R^{(a b)}{ }_{c d} .
$$

Note also that, from $g^{a b} g_{b c}=\delta_{c}^{a}$, it follows that

$$
D_{c} g_{a b}=-\Xi_{a b c}
$$

Contracting the last of (3.17) with $g_{a b}$ gives

$$
S_{c d}=g_{a b} D_{[c} \Xi_{d]}^{a b}+\frac{1}{2} \Xi_{a f}^{a} T_{c d}^{f},
$$

while (3.14) contracted with $g^{b f}$ gives

$$
\begin{aligned}
D^{b} R_{b a}-\frac{1}{2} D_{a} R=\frac{1}{2} R^{b c}{ }_{d a} T_{b c}^{d} & +R^{b}{ }_{c} T_{a b}^{c} \\
& -\frac{1}{2}\left(\Xi^{b c}{ }_{c} R_{b a}+\Xi^{c d}{ }_{b} R^{b}{ }_{c d a}+\Xi^{b c}{ }_{a} R_{b c}\right) \\
+ & D_{c}\left(D_{[b} \Xi^{b c}{ }_{a]}+\frac{1}{2} \Xi^{b c}{ }_{d} T^{d}{ }_{b a}\right)+\left(D_{[b} \Xi^{b c}{ }_{d]}+\frac{1}{2} \Xi^{b c}{ }_{d} T_{b d}\right) T^{d}{ }_{a c} .
\end{aligned}
$$

The curvature scalar is defined by $R=g^{a b} R_{a b}$, the Einstein tensor by

$$
G_{a b}=R_{(a b)}-\frac{1}{2} g_{a b} R
$$


When combining with (3.13), the contracted Bianchi identity (3.20) written in terms of the Einstein tensor is

$$
\begin{aligned}
D^{b} G_{b a}=\frac{1}{2} R^{b c}{ }_{d a} T_{b c}^{d}+ & R_{c}^{b} T_{a b}^{c}-\frac{1}{2} \Xi_{a b}{ }^{b} R \\
+ & \frac{1}{2} D^{b}\left(S_{a b}-D_{c} T_{a b}^{c}-2 D_{[a} T_{b] c}^{c}-T^{c}{ }_{d c} T_{a b}^{d}\right) \\
& \quad-\frac{1}{2}\left(\Xi^{b c}{ }_{c} R_{b a}+\Xi^{c d}{ }_{b} R^{b}{ }_{c d a}+\Xi^{b c}{ }_{a} R_{b c}\right) \\
& +D_{c}\left(D_{[b} \Xi^{b c}{ }_{a]}+\frac{1}{2} \Xi^{b c}{ }_{d} T_{b a}^{d}\right)+\left(D_{[b} \Xi_{d]}^{b c}+\frac{1}{2} \Xi_{d}^{b c} T_{b d}^{d}\right) T_{a c}^{d} .
\end{aligned}
$$

By the usual manipulations, one may show in full generality that the existence of the metric implies that the most general connection can be written as

$$
\Gamma_{a b c}=\{a b c\}+M_{a b c}+K_{a b c}+r_{a b c},
$$

where

$$
\begin{gathered}
\{a b c\}=\frac{1}{2}\left(g_{a b, c}+g_{a c, b}-g_{b c, a}\right)=\{a c b\}, \\
M_{a b c}=\frac{1}{2}\left(\Xi_{a b c}+\Xi_{a c b}-\Xi_{b c a}\right)=M_{a c b}, \\
K_{a b c}=\frac{1}{2}\left(T_{b a c}+T_{c a b}-T_{a b c}\right)=-K_{b a c}, \\
r_{a b c}=\frac{1}{2}\left(D_{b a c}+D_{c a b}-D_{a b c}\right)=-r_{b a c} .
\end{gathered}
$$

Furthermore, one can directly show that

$$
\Gamma_{b \mu}^{a}=e^{a}{ }_{v}\left(\partial_{\mu} e_{b}{ }^{v}+\Gamma_{\rho \mu}^{v} e_{b}^{\rho}\right) \Longleftrightarrow \Gamma_{a b c}=e_{a v} \partial_{c} e_{b}{ }^{v}+e_{a}{ }^{\mu} e_{b}{ }^{v} e_{c}{ }^{\rho} \Gamma_{\mu v \rho} .
$$

Finally, we will need the following variation

$$
\delta R_{b \mu \nu}^{a}=D_{\mu} \delta \Gamma_{b v}^{a}-D_{\nu} \delta \Gamma_{b \mu}^{a} .
$$

\subsection{Coordinate basis, torsionless connection}

We now consider the particular case of a coordinate basis, $e_{a}{ }^{\mu}=\delta_{a}{ }^{\mu}$ so that $D^{\lambda}{ }_{\mu \nu}=0$ and $T_{\mu \nu}^{\lambda}=\Gamma_{v \mu}^{\lambda}-\Gamma_{\mu v}^{\lambda}$. We also impose vanishing of torsion, which requires the connection to be symmetric, $\Gamma_{\mu \nu}^{\lambda}=\Gamma_{\nu \mu}^{\lambda}$. In this case, equation (3.13) implies $S_{\mu \nu}=R_{\mu \nu}-R_{v \mu}$ and the contracted Bianchi identities (3.22) become

$$
D^{v} G_{v \mu}=D^{v} R_{[\mu v]}+D_{\lambda} R^{(\lambda v)}{ }_{v \mu}-\frac{1}{2}\left(D_{v} g^{v \lambda} R_{\lambda \mu}+D_{v} g^{\lambda \rho} R_{\lambda \rho \mu}^{v}+D_{\mu} g^{v \lambda} R_{v \lambda}+D^{v} g_{\mu v} R\right),
$$

while the variation (3.29) simplifies to

$$
\delta R_{\beta \mu v}^{\alpha}=D_{\mu} \delta \Gamma_{\beta v}^{\alpha}-D_{v} \delta \Gamma_{\beta \mu}^{\alpha} .
$$

We also have

$$
\partial_{\mu}\left(\sqrt{|g|} v^{\mu}\right)=\sqrt{|g|}\left(D_{\mu}-\Gamma_{\mu \nu}^{v}+\frac{1}{2} g^{v \lambda} \partial_{\mu} g_{v \lambda}\right) \nu^{\mu}=D_{\mu}\left(\sqrt{|g|}{ }^{\mu}\right),
$$


where the last equality follows by introducing the convenient definition for the covariant derivative of a scalar density,

$$
D_{\mu} \sqrt{|g|}=\sqrt{|g|}\left(\frac{1}{2} g^{\nu \lambda} \partial_{\mu} g_{\nu \lambda}-\Gamma_{\mu \nu}^{v}\right) .
$$

If in addition, as will be imposed below on-shell, one requires metricity, $\Xi^{a b}=d g^{a b}+2 \Gamma^{(a b)}=$ 0 , one recovers the standard Christoffel connection

$$
\Gamma_{\lambda \mu v}=\frac{1}{2}\left(\partial_{v} g_{\lambda \mu}+\partial_{\mu} g_{\lambda v}-\partial_{\lambda} g_{\mu v}\right)
$$

The contracted Bianchi identities (3.30) reduce to

$$
D^{v} G_{v \mu}=0
$$

and (3.32) to

$$
\partial_{\mu}\left(\sqrt{|g|} v^{\mu}\right)=\sqrt{|g|} D_{\mu} v^{\mu}
$$

\section{Palatini formulation}

\subsection{Variational principle}

In the formulation discussed for example in [7], one uses a coordinate basis $e_{a}^{\mu}=\delta_{a}^{\mu}, D_{v \rho}^{\mu}=$ 0 with a metric $g_{\mu \nu}$ and a torsionfree connection $\Gamma_{\mu \nu}^{\lambda}=\Gamma_{v \mu}^{\lambda}$ as variables ${ }^{1}$ to write the Palatini action as

$$
S^{P}\left[g_{\mu \nu}, \Gamma_{\mu \nu}^{\lambda}\right]=\kappa \int d^{n} x L^{P}=\kappa \int d^{n} x \sqrt{|g|}(R-2 \Lambda),
$$

where $\kappa^{-1}=16 \pi G$ and we assume $n \geq 3$. Using (3.31), the variation of the action is given by

$$
\delta S^{P}=\kappa \int d^{n} x \sqrt{|g|}\left[-\left(G^{\mu v}+\Lambda g^{\mu v}\right) \delta g_{\mu v}+g^{\alpha \beta}\left(D_{\mu} \delta \Gamma_{\alpha \beta}^{\mu}-D_{\beta} \delta \Gamma_{\alpha \mu}^{\mu}\right)\right] .
$$

Using in addition (3.32) and neglecting boundary terms yields

$$
\begin{aligned}
\delta S^{P}=\kappa \int d^{n} x\left[-\sqrt{|g|}\left(G^{\mu v}+\Lambda g^{\mu v}\right)\right. & \delta g_{\mu v} \\
& \left.+\left(-D_{\mu}\left[\sqrt{|g|} g^{\alpha \beta}\right]+D_{\lambda}\left[\sqrt{|g|} g^{\alpha \lambda} \delta_{\mu}^{\beta}\right]\right) \delta \Gamma^{\mu}{ }_{\alpha \beta}\right],
\end{aligned}
$$

so that the Euler-Lagrange derivatives of $L^{P}$ with respect to the fields $g_{\mu \nu}$ and $\Gamma_{\mu \nu}^{\lambda}$ take the form

$$
\begin{aligned}
\frac{\delta L^{P}}{\delta g_{\mu v}} & =-\sqrt{|g|}\left(G^{\mu v}+\Lambda g^{\mu v}\right), \\
\frac{\delta L^{P}}{\delta \Gamma^{\mu}{ }_{\alpha \beta}} & =-D_{\mu}\left[\sqrt{|g|} g^{\alpha \beta}\right]+\frac{1}{2} D_{\lambda}\left[\sqrt{|g|} g^{\alpha \lambda} \delta_{\mu}^{\beta}\right]+\frac{1}{2} D_{\lambda}\left[\sqrt{|g|} g^{\beta \lambda} \delta_{\mu}^{\alpha}\right] .
\end{aligned}
$$

Contracting the equations of motion corresponding to (4.5) with $\delta_{\beta}^{\mu}$ gives $D_{\beta}\left[\sqrt{|g|} g^{\alpha \beta}\right]=0$. When re-injecting this result into the equation of motion, this implies $D_{\mu}\left[\sqrt{|g|} g^{\alpha \beta}\right]=0$. From $\operatorname{det}\left(\sqrt{|g|} g^{\alpha \beta}\right)=|g|^{\frac{n-2}{2}}$, one then deduces that

$\delta|g|^{\frac{1}{2}}=\delta\left(\operatorname{det}\left(\sqrt{|g|} g^{\alpha \beta}\right)\right)^{\frac{1}{n-2}}=\frac{1}{n-2} \operatorname{det}\left(\sqrt{|g|} g^{\alpha \beta}\right)^{\frac{1}{n-2}-1} \delta \operatorname{det}\left(\sqrt{|g|} g^{\alpha \beta}\right)=\frac{1}{n-2} g_{\alpha \beta} \delta\left(\sqrt{|g|} g^{\alpha \beta}\right)$.

\footnotetext{
${ }^{1}$ Adapting the arguments below to the case where the variables are chosen as the contravariant metric tensor density and the connection as done in $[1,2]$ is straightforward.
} 
When the variation corresponds to the covariant derivative $D_{\mu}$, we deduce that these equations of motion imply that $D_{\mu} \sqrt{|g|}=0$, and then metricity, $D_{\mu} g^{\alpha \beta}=0$. Since this implies (3.34), it follows that $\Gamma_{\alpha \beta}^{\mu}$ are auxiliary fields, i.e., fields that can be eliminated algebraically by their own equations of motion.

\subsection{Gauge symmetries and Noether identities}

If $\xi^{\mu}(x)$ denotes the vector field parametrizing an infinitesimal diffeomorphism, the variation of the variables of the variational principle at a given point is

$$
\begin{aligned}
\delta_{\xi} g_{\mu v} & =\mathscr{L}_{\xi} g_{\mu v}=\xi^{\rho} \partial_{\rho} g_{\mu v}+g_{\rho v} \partial_{\mu} \xi^{\rho}+g_{\mu \rho} \partial_{v} \xi^{\rho} \\
\delta_{\xi} \Gamma^{\mu}{ }_{v \rho} & =\partial_{\rho} \partial_{v} \xi^{\mu}+\xi^{\sigma} \partial_{\sigma} \Gamma^{\mu}{ }_{v \rho}-\partial_{\sigma} \xi^{\mu} \Gamma^{\sigma}{ }_{v \rho}+\partial_{v} \xi^{\sigma} \Gamma^{\mu}{ }_{\sigma \rho}+\partial_{\rho} \xi^{\sigma} \Gamma^{\mu}{ }_{v \sigma} .
\end{aligned}
$$

These transformations are infinitesimal gauge symmetries of the Palatani formulation in the sense that $\delta_{\xi} L^{P}=\partial_{\mu}\left(\xi^{\mu} L^{P}\right)$ for all $\xi^{\mu}(x)$. As usual, this follows as a consequence of the fact that $L^{P}$ transforms like a scalar density under finite diffeomorphisms.

At this stage, we note that the transformation law of the connection in (4.7) involves derivatives of the gauge parameter $\xi^{\mu}$ up to second order. Therefore, even though the Euler-Lagrange equations (4.4) and (4.5) are of first order, the theory is not in the class of first order theories described for instance [24].

The Noether identities and the weakly vanishing Noether current associated to these gauge symmetries are then identified by using Leibniz rule to write the analog of (2.1) in the present case,

$$
\frac{\delta \kappa L^{P}}{\delta g_{\mu \nu}} \delta_{\xi} g_{\mu v}+\frac{\delta \kappa L^{P}}{\delta \Gamma_{\alpha \beta}^{\mu}} \delta_{\xi} \Gamma_{\alpha \beta}^{\mu}=\xi^{\rho} N_{\rho}+\partial_{\mu} S_{\xi}^{\mu}
$$

which leads to the Noether identities

$$
\begin{aligned}
& \kappa^{-1} N_{\rho}=\frac{\delta L^{P}}{\delta g_{\mu \nu}} \partial_{\rho} g_{\mu v}-2 \partial_{\sigma}\left(\frac{\delta L^{P}}{\delta g_{\sigma v}} g_{\rho v}\right) \\
& \quad+\partial_{\alpha} \partial_{\beta}\left(\frac{\delta L^{P}}{\delta \Gamma^{\rho}{ }_{\alpha \beta}}\right)+\frac{\delta L^{P}}{\delta \Gamma^{\mu}{ }_{\alpha \beta}} \partial_{\rho} \Gamma_{\alpha \beta}^{\mu}+\partial_{\sigma}\left(\frac{\delta L^{P}}{\delta \Gamma_{\alpha \beta}} \Gamma_{\alpha \beta}^{\sigma}\right)-2 \partial_{\sigma}\left(\frac{\delta L^{P}}{\delta \Gamma^{\mu}{ }_{\sigma \beta}} \Gamma_{\rho \beta}^{\mu}\right)=0 .
\end{aligned}
$$

These identities correspond to the contracted Bianchi identities (3.30). Indeed, (4.9) can be rewritten as

$$
\frac{\delta L^{P}}{\delta g_{\mu \nu}} D_{\rho} g_{\mu \nu}-2 D_{\mu}\left(g_{\rho v} \frac{\delta L^{P}}{\delta g_{\mu \nu}}\right)+\frac{\delta L^{P}}{\delta \Gamma^{\tau} \sigma v} R_{\sigma \rho v}^{\tau}+D_{\sigma} D_{v}\left(\frac{\delta L^{P}}{\delta \Gamma_{\sigma v}{ }^{\prime}}\right)=0 .
$$

By inserting (4.4) and (4.5) into (4.10), one recovers (3.30). For this computation, the identities

$$
\begin{gathered}
D_{\mu} \sqrt{|g|}=\frac{1}{2} \sqrt{|g|} g^{\alpha \beta} D_{\mu} g_{\alpha \beta}, \quad\left[D_{\mu}, D_{v}\right] \sqrt{|g|}=-\sqrt{|g|}\left(R_{\mu v}-R_{v \mu}\right), \\
{\left[D_{\mu}, D_{v}\right] D_{\lambda} \sqrt{|g|}=-D_{\tau} \sqrt{|g|} R_{\lambda \mu \nu}^{\tau}-D_{\lambda} \sqrt{|g|}\left(R_{\mu v}-R_{v \mu}\right)}
\end{gathered}
$$

are useful. 


\subsection{Construction of the co-dimension 2 form}

We also get from (4.8) the weakly vanishing Noether current associated with the gauge symmetries,

$$
\kappa^{-1} S_{\xi}^{\mu}=2 \frac{\delta L^{P}}{\delta g_{\mu \tau}} \xi_{\tau}+2 \frac{\delta L^{P}}{\delta \Gamma^{\tau} \mu \rho} D_{\rho} \xi^{\tau}-\Gamma_{\rho \sigma}^{\mu} \frac{\delta L^{P}}{\delta \Gamma^{\tau} \sigma \rho} \xi^{\tau}-\partial_{\rho}\left(\frac{\delta L^{P}}{\delta \Gamma^{\tau} \mu \rho} \xi^{\tau}\right)
$$

In order to compute the co-dimension 2 forms, one now needs to insert this expression into the general formula (2.7). Note that (4.13) involves second order derivatives of the fields in the last term as a consequence of the second order derivatives on the gauge parameters. Since these second derivatives occur under a total derivative however, the properties (2.9) allow one to reduce the actual computation to one involving only the Euler-Lagrange derivatives of order one acting on expressions which are at most of first order in derivatives,

$$
\begin{aligned}
\kappa^{-1} k_{\xi}^{[\mu v]}=\frac{1}{2} \delta \phi^{i} \frac{\delta}{\delta \phi_{v}^{i}}\left[2 \frac{\delta L^{P}}{\delta g_{\mu \tau}} \xi_{\tau}+2 \frac{\delta L^{P}}{\delta \Gamma^{\tau} \mu \rho} D_{\rho} \xi^{\tau}-\Gamma^{\mu}{ }_{\rho \sigma} \frac{\delta L^{P}}{\delta \Gamma^{\tau} \sigma \rho} \xi^{\tau}\right] \\
-\frac{1}{3} \partial_{\rho}\left[\delta \phi^{i} \frac{\delta}{\delta \phi_{v}^{i}}\left(\frac{\delta L^{P}}{\delta \Gamma^{\tau} \mu \rho} \xi^{\tau}\right)\right]-(\mu \leftrightarrow v) .
\end{aligned}
$$

To proceed in this computation, let us introduce the notations $h_{\mu v}=\delta g_{\mu v}, \delta \Gamma^{\rho}{ }_{\mu \nu}=C^{\rho}{ }_{\mu \nu}$, indices being lowered and raised with $g_{\mu \nu}$ and its inverse, and $h=h_{\mu}^{\mu}$. Using

$$
\begin{aligned}
& \delta\left(\sqrt{|g|} g^{\mu \lambda}\right)=-\sqrt{|g|} h^{\mu \lambda}+\frac{1}{2} \sqrt{|g|} g^{\mu \lambda} h, \\
& \partial_{\lambda}\left(\sqrt{|g|} h^{\mu \lambda} \xi^{v}\right)=D_{\lambda}\left(\sqrt{|g|} h^{\mu \lambda} \xi^{v}\right)-\sqrt{|g|} \Gamma^{\mu} \lambda \tau h^{\tau \lambda} \xi^{v}-\sqrt{|g|} \Gamma^{v} \lambda \tau h^{\mu \lambda} \xi^{\tau},
\end{aligned}
$$

one finally obtains the explicit expression for the co-dimension 2 form,

$$
\begin{aligned}
& \kappa^{-1} k_{\xi}^{[\mu v]}=\sqrt{|g|}\left[\xi^{\sigma} C_{\sigma}^{\mu \nu}-\xi^{\mu} C_{\sigma}^{\sigma}{ }^{v}+\frac{1}{2} \xi^{\mu} C_{\sigma}^{v \sigma}-h^{v \sigma} D_{\sigma} \xi^{\mu}+\frac{1}{2} h D^{v} \xi^{\mu}\right. \\
& \left.\quad-\frac{1}{2} \xi^{v} D_{\sigma} h^{\mu \sigma}+\frac{1}{4} \xi^{v} D^{\mu} h\right]-\frac{1}{2} h^{\mu \lambda} \xi^{v} D_{\lambda}(\sqrt{|g|})+\frac{1}{4} h \xi^{v} D_{\lambda}\left(\sqrt{|g|} g^{\mu \lambda}\right)-(\mu \leftrightarrow v) .
\end{aligned}
$$

The breaking term is easy to work out since it merely involves the Euler-Lagrange derivatives of order one acting on expressions that are at most of first order in derivatives. It is given by

$$
\kappa^{-1} b^{\mu}=\delta_{\xi} \Gamma_{\rho \nu}^{\mu} \delta\left(\sqrt{|g|} g^{v \rho}\right)-\delta_{\xi} \Gamma_{\rho \nu}^{\nu} \delta\left(\sqrt{|g|} g^{\mu \rho}\right)-\left(\delta_{\xi} \leftrightarrow \delta\right) .
$$

\subsection{Reduction to the metric formulation}

We now compare the expression (4.15) with the standard results obtained in the metric formulation, where absence of torsion and metricity are assumed. For this purpose, let us go on-shell for the auxiliary fields $\Gamma^{\rho}{ }_{\mu \nu}$ appearing in the co-dimension 2 form (4.15). One directly gets

$$
\begin{gathered}
\kappa^{-1} k_{\xi}^{[\mu v]}=\sqrt{|g|}\left[\xi^{\sigma} C_{\sigma}^{\mu v}-\xi^{\mu} C_{\sigma}^{\sigma}{ }^{v}+\frac{1}{2} \xi^{\mu} C_{\sigma}^{v \sigma}-\frac{1}{2} \xi^{v} D_{\sigma} h^{\mu \sigma}+\frac{1}{4} \xi^{v} D^{\mu} h\right. \\
\left.-h^{v \sigma} D_{\sigma} \xi^{\mu}+\frac{1}{2} h D^{v} \xi^{\mu}\right]-(\mu \leftrightarrow v) .
\end{gathered}
$$


When taking into account that $D_{\mu}$ is now the connection involving the Christoffel symbols, so that $C_{\tau \sigma}^{\mu}=\frac{1}{2}\left(D_{\tau} h_{\sigma}^{\mu}+D_{\sigma} h_{\tau}^{\mu}-D^{\mu} h_{\tau \sigma}\right)$, we obtain

$$
\kappa^{-1} k_{\xi}^{[\mu v]}=\sqrt{|g|}\left[\xi_{\sigma} D^{v} h^{\mu \sigma}+\xi^{v} D^{\mu} h-\xi^{v} D_{\sigma} h^{\mu \sigma}-h^{v \sigma} D_{\sigma} \xi^{\mu}+\frac{1}{2} h D^{v} \xi^{\mu}\right]-(\mu \leftrightarrow v) .
$$

Assuming that $\xi^{\mu}$ is a Killing vector, namely $D_{\sigma} \xi^{\mu}+D^{\mu} \xi_{\sigma}=0$, this expression reproduces exactly the co-dimension 2 form obtained in metric formalism [20,32,33]. Therefore, we see that the co-dimension 2 forms expressions are equivalent in Palatini and metric formalisms for exact reducibility parameters. However, this result does not hold when $\xi^{\mu}$ is not a Killing vector. In particular, the expressions may not match when using asymptotic Killing vectors, as previously pointed out in the Cartan formulation of general relativity [19,23].

\section{Acknowledgements}

This work is supported by the F.R.S.-FNRS Belgium through conventions FRFC PDR T.1025.14 and IISN 4.4503.15. The work of P. Mao is supported in part by the National Natural Science Foundation of China under Grant Nos. 11905156 and 11935009 . The work of R. Ruzziconi is supported by a FRIA fellowship.

\section{References}

[1] R. Arnowitt and S. Deser, "Quantum Theory of Gravitation: General Formulation and Linearized Theory," Phys. Rev. 113 (1959) 745-750.

[2] R. L. Arnowitt, S. Deser, and C. W. Misner, "Dynamical Structure and Definition of Energy in General Relativity," Phys. Rev. 116 (1959) 1322-1330.

[3] R. Arnowitt, S. Deser, and C. W. Misner, “Canonical variables for general relativity," Phys. Rev. 117 no. 6, (Mar, 1960) 1595-1602.

[4] R. Arnowitt, S. Deser, and C. W. Misner, "Energy and the criteria for radiation in general relativity," Phys. Rev. 118 (May, 1960) 1100-1104.

[5] R. Arnowitt, S. Deser, and C. W. Misner, "Coordinate invariance and energy expressions in general relativity,” Phys. Rev. 122 (1961) 997-1006.

[6] R. Arnowitt, S. Deser, and C. Misner, Gravitation, an Introduction to Current Research, ch. 7. The Dynamics of General Relativity, pp. 227-265. Wiley, New York, 1962.

[7] C. Misner, K. Thorne, and J. Wheeler, Gravitation. W.H. Freeman, New York, 1973.

[8] F. W. Hehl, J. D. McCrea, E. W. Mielke, and Y. Neeman, "Metric affine gauge theory of gravity: Field equations, noether identities, world spinors, and breaking of dilation invariance," Phys. Rept. 258 (1995) 1-171, gr-qc/9402012.

[9] B. Julia and S. Silva, "Currents and superpotentials in classical gauge invariant theories. 1. Local results with applications to perfect fluids and general relativity," Class.Quant.Grav. 15 (1998) 2173-2215, gr-qc/9804029.

[10] B. Julia and S. Silva, "Currents and superpotentials in classical gauge theories. II: Global aspects and the example of affine gravity," Class. Quant. Grav. 17 (2000) 4733-4744, gr-qc/ 0005127. 
[11] B. Julia and S. Silva, "On covariant phase space methods," hep-th/0205072.

[12] A. Ashtekar, J. Engle, and D. Sloan, “Asymptotics and Hamiltonians in a First order formalism," Class. Quant. Grav. 25 (2008) 095020, arXiv:0802.2527 [gr-qC] .

[13] T. Jacobson and A. Mohd, "Black hole entropy and Lorentz-diffeomorphism Noether charge," Phys. Rev. D92 (2015) 124010, arXiv: 1507.01054 [gr-qc] .

[14] A. Corichi, I. Rubalcava, and T. Vukasinac, "Hamiltonian and Noether charges in first order gravity," Gen. Rel. Grav. 46 (2014) 1813, arXiv:1312.7828 [gr-qc] .

[15] L. Lehner, R. C. Myers, E. Poisson, and R. D. Sorkin, "Gravitational action with null boundaries," Phys. Rev. D94 no. 8, (2016) 084046, arXiv:1609.00207 [hep-th] .

[16] Y. Korovin, "Asymptotic symmetries and geometry on the boundary in the first order formalism," JHEP 03 (2018) 017, arXiv:1709.07647 [hep-th] .

[17] E. De Paoli and S. Speziale, "A gauge-invariant symplectic potential for tetrad general relativity," JHEP 07 (2018) 040, arXiv:1804.09685 [gr-qC] .

[18] E. Frodden and D. Hidalgo, "Surface Charges Toolkit for Gravity," arXiv: 1911.07264 [hep-th].

[19] R. Oliveri and S. Speziale, "Boundary effects in general relativity with tetrad variables," arXiv:1912.01016 [gr-qC].

[20] G. Barnich and F. Brandt, "Covariant theory of asymptotic symmetries, conservation laws and central charges,” Nucl. Phys. B633 (2002) 3-82, hep-th/ 0111246.

[21] G. Barnich, "Boundary charges in gauge theories: Using Stokes theorem in the bulk," Class. Quant. Grav. 20 (2003) 3685-3698, hep-th/ 0301039.

[22] G. Barnich and G. Compère, "Surface charge algebra in gauge theories and thermodynamic integrability," J. Math. Phys. 49 (2008) 042901, arXiv:0708.2378 [gr-qC] .

[23] G. Barnich, P. Mao, and R. Ruzziconi, "Conserved currents in the Cartan formulation of general relativity," in About Various Kinds of Interactions: Workshop in honour of Professor Philippe Spindel. 2016. arXiv:1611.01777 [gr-qC].

[24] G. Barnich, P. Mao, and R. Ruzziconi, "BMS current algebra in the context of the Newman-Penrose formalism," Class. Quant. Grav. 37 no. 9, (2020) 095010, arXiv:1910.14588 [gr-qc] .

[25] Y. N. Obukhov, F. Portales-Oliva, D. Puetzfeld, and G. F. Rubilar, "Invariant conserved currents in generalized gravity," Phys. Rev. D 92 no. 10, (2015) 104010, arXiv:1507.02191 [gr-qC].

[26] M. Henneaux and C. Teitelboim, Quantization of Gauge Systems. Princeton University Press, 1992.

[27] I. Anderson, "The variational bicomplex,” tech. rep., Formal Geometry and Mathematical Physics, Department of Mathematics, Utah State University, 1989.

[28] P. Olver, Applications of Lie Groups to Differential Equations. Springer Verlag, New York, 2nd ed., 1993. 1st ed., 1986.

[29] R. M. Wald and A. Zoupas, "A general definition of conserved quantities in general relativity and other theories of gravity," Phys. Rev. D61 (2000) 084027, gr-qc/ 9911095.

[30] G. Barnich and C. Troessaert, "BMS charge algebra," JHEP 1112 (2011) 105, arXiv: 1106.0213 [hep-th]. 
[31] G. Barnich and C. Troessaert, "Comments on holographic current algebras and asymptotically flat four dimensional spacetimes at null infinity," JHEP 1311 (2013) 003, arXiv: 1309.0794

[hep-th].

[32] V. Iyer and R. M. Wald, "Some properties of Noether charge and a proposal for dynamical black hole entropy," Phys. Rev. D50 (1994) 846-864, gr-qc/ 9403028.

[33] I. M. Anderson and C. G. Torre, "Asymptotic conservation laws in field theory," Phys. Rev. Lett. 77 (1996) 4109-4113, hep-th/9608008. 